\title{
Coexisting Mucinous Cystic Neoplasm of the Pancreas and Type 1 Autoimmune Pancreatitis
}

Mee-Jeong Kim · Tae Jun Song ${ }^{1}$ Hyoung Jung $\mathrm{Kim}^{2} \cdot$ Song-Cheol Kim ${ }^{3}$ Myung-Hwan Kim ${ }^{1}$ Seung-Mo Hong

Departments of Pathology and ${ }^{1}$ Gastroenterology, ${ }^{2}$ Department of Radiology and Research Institute of Radiology, ${ }^{3}$ Department of Surgery, Asan Medical Center, University of Ulsan College of Medicine, Seoul, Korea

Received: October 1, 2018

Revised: October 17, 2018

Accepted: October 24, 2018

\section{Corresponding Author}

Seung-Mo Hong, MD, PhD

Department of Pathology, Asan Medical Center,

University of Ulsan College of Medicine,

88 Olympic-ro 43-gil, Songpa-gu, Seoul 05505

Korea

Tel: +82-2-3010-4558

Fax: $+82-2-472-7898$

E-mail:smhong28@gmail.com
Type 1 autoimmune pancreatitis (AIP1) is an IgG4-related systemic disease that mimics tumors. We report a rare case of AIP1 accompanied by mucinous cystic neoplasm (MCN). A pancreatic lesion was incidentally detected in a woman in her 60s. After 6 years of follow-up, the lesion abruptly increased in size. Computed tomography showed a $3.5 \mathrm{~cm}$ unilocular cyst in the tail of the pancreas and distal pancreatectomy was performed. On microscopic examination, the cyst was lined by mucinous and non-mucinous epithelial cells with mild cytologic atypia. The surrounding stroma comprised ovarian-type spindle cells with progesterone receptor positivity. The pericystic pancreas exhibited multifocal lymphoid follicles, lymphoplasmacytic infiltrations, obliterative phlebitis, and storiform fibrosis. IgG4-positive plasma cell infiltration ( 215 cells high-power field) and the IgG4/lgG ratio (57\%) were increased. Cases of MCN coexisting with AIP1 are extremely rare; only two such cases have been reported in the English-language literature. This third case featured low-grade MCN with AIP1.

Key Words: Pancreas; Mucinous cystic neoplasm; Autoimmune pancreatitis
Mucinous cystic neoplasms (MCNs) of the pancreas are cystforming and composed of columnar mucinous epithelia with varying degrees of cytologic atypia. ${ }^{1}$ Traditionally, MCNs have been classified as low-, intermediate-, or high-grade dysplasia according to the degree of cytological atypia. ${ }^{1}$ Recently, however, the Baltimore consensus meeting proposed a two-tiered system for pancreatic precursor lesions, including MCNs and intraductal papillary mucinous neoplasms (IPMNs), classifying them as either low-grade (which includes the previous low- and intermediategrade dysplasia) or high-grade. ${ }^{2} \mathrm{MCNs}$ are histologically recognizable precursor lesions of pancreatic ductal adenocarcinomas. ${ }^{3}$ They are associated with ovarian-type stroma and do not communicate with the pancreatic ductal system, which are diagnostic differential points with IPMNs. ${ }^{4}$ The majority of MCNs occur in women aged 40-60 years at the time of diagnosis. ${ }^{1}$ The body and tail of the pancreas are the most common locations for MCNs; involvement of the head is rare. ${ }^{1}$

Autoimmune pancreatitis (AIP) is a type of chronic pancre- atitis that presents with obstructive jaundice and/or a pancreatic mass, with histologic features of dense lymphoplasmacytic infiltrations and fibrosis; it responds well to steroid treatment. ${ }^{5}$ AIP is classified as types 1 and 2 . The diagnostic criteria for type 1 AIP (AIP1) are well established by international consensus and include clinical, serological, imaging, and pathology components. ${ }^{6}$ The characteristic histologic features of AIP1 are dense lymphoplasmacytic infiltration, storiform fibrosis, and obliterative phlebitis. ${ }^{7}$ A diagnosis of AIP1 is made based on the number of these histologic features observed and the number of IgG4-positive plasma cell infiltrations seen in high-power fields ( $>50$ cells for surgical resection or $>10$ cells for biopsy specimens). ${ }^{?}$

There have been several case reports about AIP1 accompanied by other pancreatic lesions, such as a pseudocyst or IPMN.$^{8-11}$ However, MCN with AIP1 is extremely rare and only two cases have been reported in the English-language literature. ${ }^{12,13}$ Here, we report a third case, a patient with both low-grade $\mathrm{MCN}$ and AIP1. 


\section{CASE REPORT}

A woman in her 60s admitted our hospital with a symptomless, incidentally detected pancreatic cystic lesion. Initial computed tomography (CT) imaging revealed a $2.2 \mathrm{~cm}$ unilocular cystic pancreas lesion in the body and tail of the pancreas without accompanying wall enhancement and with no mural nodule. There was no evidence of narrowing of the main pancreatic duct or of acute pancreatitis (Fig. 1A). The initial laboratory findings were unremarkable, including normal levels of $\gamma$-GT (13 IU/L; normal range, 5 to $36 \mathrm{IU} / \mathrm{L}$ ), amylase (65 U/L; normal range, 30 to $110 \mathrm{U} / \mathrm{L}$ ), and lipase ( $37 \mathrm{U} / \mathrm{L}$; normal range, 13 to $60 \mathrm{U} /$ L). Suspecting either a pseudocyst or MCN, the patient was regularly followed up. After 6 years of follow-up, the cystic lesion had increased in size to $3.5 \mathrm{~cm}$ (Fig. 1B). As a result, the patient underwent a distal pancreatectomy. On gross examination, a 3.5 $\mathrm{cm}$-sized unilocular cyst was observed in the body and tail of the pancreas. The cyst wall was irregularly thickened, firm, and fibrotic (Fig. 1C). The inner surface of the cyst was yellowish white and rough, with multifocal hemorrhagic spots. The cyst contained sticky mucinous fluid. The remaining pancreatic parenchyma away from the cystic neoplasm was unremarkable, and there was no dilatation of the main pancreatic duct.

Low-power magnification images revealed that the cystic wall was surrounded by dense spindle cell clusters, with numerous lymphoid follicles observed in a fibrotic background (Fig. 1D). The thin sections of the cyst wall were lined by a single layer of epithelial cells that were mostly non-mucinous or focally mucinous (Fig. 1E). The epithelial cells were pseudostratified without showing pleomorphism or prominent nucleoli, and the surrounding dense spindle cells were closely packed, showing ovariantype spindle cells. Nuclear progesterone receptor labeling showed these spindle cells to be dense (Fig. 1F). The irregularly thickened portions of the cyst wall observed in the gross examination contained multiple lymphoid follicles in a background of chronic inflammatory cell infiltrations, predominantly lymphoplasmacytic (Fig. 1G). Some areas contained dense periductal lymphoplasmacytic infiltrations (Fig. 1H). Several foci of obliterative
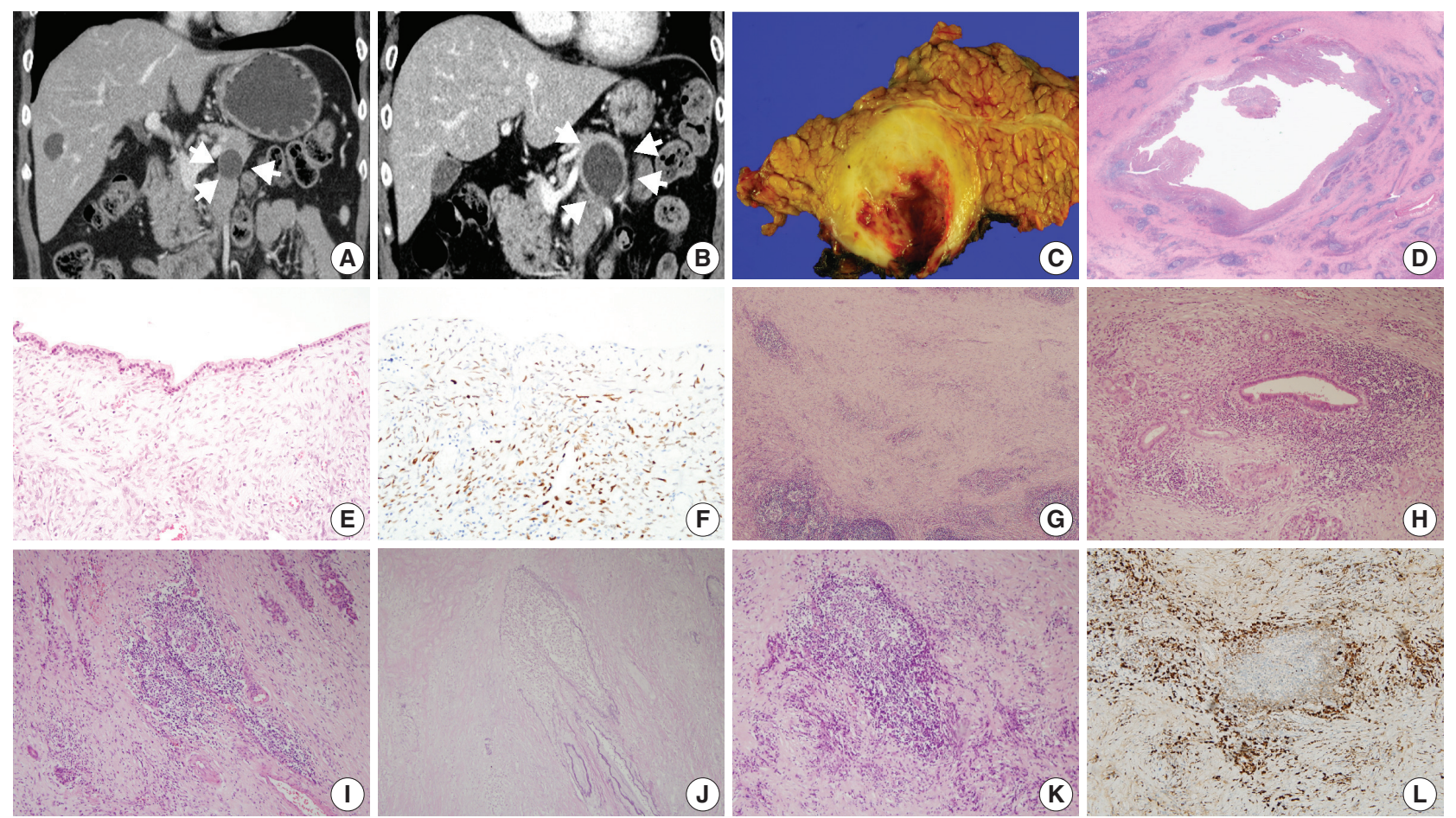

Fig. 1. (A) Coronal contrast-enhanced computed tomography image showing a unilocular cyst in the pancreas tail. (B) Six years later, the cyst had grown, with its longest diameter increasing from 2.2 to $3.5 \mathrm{~cm}$, as well as thickening of the cyst wall (arrowheads). (C) The unilocular cyst in the body and tail of the pancreas showing irregular thickening of the cyst wall. (D) Scanning power image showing lymphoid follicles and lymphoplasmacytic infiltration around the cyst wall. (E) The cyst wall was lined mostly by non-mucinous and focally mucinous epithelial cells with mild cytologic atypia. (F) Progesterone receptor nuclear labeling highlighted ovarian-type stromal cells. (G) The irregularly thickened cyst wall contained multiple lymphoid follicles in a background of chronic inflammatory cell infiltrations. (H) Some areas showed dense periductal lymphoplasmacytic infiltrations. (I, J) Several foci of obliterative phlebitis were noted by hematoxylin and eosin staining (I) and elastic staining (J). (K, L) Areas of dense lymphoplasmacytic infiltrations (K) showed numerous lgG4-positive plasma cell infiltrations $(\mathrm{L})$. 
Table 1. Comparisons of clinicopathologic characteristics of MCN in association with AIP1 with previously reported cases

\begin{tabular}{|c|c|c|c|c|c|c|c|c|}
\hline Study & Age (yr) & Sex & Dysplasia & Fibrosis & Obliterative phlebitis & IgG4+plasma cells/HPF & $\lg G 4 / \operatorname{lgG}(\%)$ & Serum lgG4 level (mg/dL) \\
\hline Yakirevich et al. ${ }^{12}$ & 33 & Female & High-grade & Storiform & Present & 75 & 42 & 97.5 \\
\hline DiCarlo et al. ${ }^{13}$ & 26 & Female & Low-grade & Dense band & Present & 160 & NA & NA \\
\hline Present case & 60 & Female & Low-grade & Storiform & Present & 215 & 57 & $0.4^{\mathrm{a}}$ \\
\hline
\end{tabular}

MCN, mucinous cystic neoplasm; AIP1, type 1 autoimmune pancreatitis; NA, not applicable; HPF, high-power field.

aSampled on postoperative day 6 .

phlebitis were revealed by $\mathrm{H} \& \mathrm{E}$ and elastic staining (Fig. 1I, J). Areas of dense lymphoplasmacytic infiltrations (Fig. 1K) included numerous IgG4-positive plasma cell infiltrations (Fig. 1L). As many as 215 IgG4-positive plasma cells were observed in the most active area. The $\operatorname{IgG} 4 / \mathrm{IgG}$ ratio was about $57 \%$. Serum IgG4 measured 6 days after the distal pancreatectomy was within the normal range $(0.4 \mathrm{mg} / \mathrm{dL})$. The patient was diagnosed with low-grade MCN associated with AIP1.

All procedures performed in the current study were approved by Institutional Review Board approval (approval No. 20181160) for waiver of the informed consent in accordance with the 1964 Helsinki declaration and its later amendments.

\section{DISCUSSION}

MCN is a precursor lesion of pancreatic ductal adenocarcinomas, whereas AIP is a tumor mimicker. This case was interesting because the patient underwent distal pancreatectomy after the $\mathrm{MCN}$ increased in size. In addition to low-grade MCN, we observed features of AIP1 in the irregularly thickened periphery of the cyst wall. This case exhibited a range of histologic features considered to be pathologic diagnostic criteria of AIP1, ${ }^{7}$ including lymphoplasmacytic infiltration, stromal storiform fibrosis, and obliterative phlebitis, along with > 50 IgG4-positive plasma cell infiltrations (with up to 215 IgG4-positive plasma cells observed). Thus, this case was histologically highly suggestive of AIP1.

However, other histologic, imaging and serologic features consistent with AIP1, such as diffuse pancreatic enlargement and pancreatic ductal strictures without significant ductal dilatation, were not observed in the present case. The patient's serum IgG4 level 6 days postoperatively was not significantly elevated.

It is rare for the histologic features of AIP1 to be reported in association with pancreatic cystic lesions, including IPMNs and pseudocysts. All the reported cases exhibited the histologic features of AIP1; in addition, some cases showed increased serum IgG4 levels, ${ }^{8,9,11}$ whereas another did not. ${ }^{10}$ There has been only one English-language report of a case of MCN accompanied by the histologic features of AIP1, and that case had an elevated serum IgG4 level. ${ }^{12}$
In the present case, the histologic features of AIP1 were observed in a limited area, the periphery of the cyst wall, and did not extend into the surrounding pancreatic parenchyma. With the normal pancreatic ductal system observed on radiologic imaging, these features can be interpreted as indicating a localized form of AIP1. Similar features were reported in the previous case. ${ }^{12}$ Comparisons of clinicopathologic characteristics of $\mathrm{MCN}$ in association with AIP1 with previously reported cases and the present case are summarized in Table 1.

The pathogenesis of AIP1 in association with MCN is not fully understood. The most plausible explanation is the co-existence of two lesions, the AIP1 and the MCN. ${ }^{13}$ Other possibilities include a secondary inflammatory response to a pre-existing $\mathrm{MCN}$ due to the use of fine needle aspiration biopsy or cytology of the MCN for histologic confirmation, or a secondary immune reaction of the periphery of the cyst wall of the pancreas to inspissated extracellular mucin from the MCN. ${ }^{12}$

In summary, we report a case of low-grade $\mathrm{MCN}$ coexisting with AIP1. This case exceptionally demonstrated the presence of both a cystic tumor and a tumor mimicker in the same pancreas.

\section{ORCID}

Mee-Jeong Kim: https://orcid.org/0000-0001-7466-7586

Tae Jun Song: https://orcid.org/0000-0002-6156-8746

Hyoung Jung Kim: https://orcid.org/0000-0003-3391-5621

Song-Cheol Kim: https://orcid.org/0000-0003-4552-4169

Myung-Hwan Kim: https://orcid.org/0000-0003-0992-6734

Seung-Mo Hong: https://orcid.org/0000-0002-8888-6007

\section{Author Contributions}

Conceptualization: SMH.

Data curation: MJK, SCK, MHK.

Formal analysis: TJS, HJK, SMH.

Investigation: MJK, SMH.

Validation: MJK, SMH.

Visualization: MJK, HJK, SMH.

Writing—original draft: MJK, SMH.

Writing—review \& editing: MJK, HJK, SMH. 


\section{Conflicts of Interest}

The authors declare that they have no potential conflicts of interest.

\section{Acknowledgments}

This study was funded by a grant (1320200) from National R\&D Program for Cancer Control, Ministry of Health \& Welfare, Republic of Korea.

\section{REFERENCES}

1. Bosman FT, Carneiro F, Hruban RH, Theise ND. WHO classification of tumours of the digestive system. 4th ed. Lyon: International Agency for Research on Cancer, 2010.

2. Basturk $\mathrm{O}$, Hong SM, Wood LD, et al. A revised classification system and recommendations from the Baltimore Consensus Meeting for Neoplastic Precursor Lesions in the Pancreas. Am J Surg Pathol 2015; 39: 1730-41.

3. Hong SM, Park JY, Hruban RH, Goggins M. Molecular signatures of pancreatic cancer. Arch Pathol Lab Med 2011; 135: 716-27.

4. Hruban RH, Pitman MB, Klimstra DS. AFIP atlas of tumor pathology, series IV. Tumors of the pancreas. Washington, DC: Armed Forces Institute of Pathology, 2007.

5. Hart PA, Zen Y, Chari ST. Recent advances in autoimmune pancreatitis. Gastroenterology 2015; 149: 39-51.
6. Shimosegawa T, Chari ST, Frulloni L, et al. International consensus diagnostic criteria for autoimmune pancreatitis: guidelines of the International Association of Pancreatology. Pancreas 2011; 40: 352-8.

7. Deshpande V, Zen Y, Chan JK, et al. Consensus statement on the pathology of IgG4-related disease. Mod Pathol 2012; 25: 1181-92.

8. Gompertz M, Morales C, Aldana H, Castillo J, Berger Z. Cystic lesions in autoimmune pancreatitis. Case Rep Gastroenterol 2015; 9: 36674

9. Nakaji S, Hirata N, Fujii H, et al. A case of focal autoimmune pancreatitis (AIP) mimicking an intraductal papillary mucinous neoplasm (IPMN). Clin J Gastroenterol 2013; 6: 329-33.

10. Urata T, Naito Y, Izumi Y, et al. Localized type 1 autoimmune pancreatitis superimposed upon preexisting intraductal papillary mucinous neoplasms. World J Gastroenterol 2013; 19: 9127-32.

11. Vaquero EC, Salcedo MT, Cuatrecasas M, et al. Autoimmune pancreatitis type-1 associated with intraduct papillary mucinous neoplasm: report of two cases. Pancreatology 2014; 14: 316-8.

12. Yakirevich E, Henriksen KJ, Miner T, Resnick MB. Mucinous cystic neoplasm of the pancreas with increased IgG4+ plasma cells and histopathologic features of autoimmune pancreatitis/IgG4-related disease. Pancreas 2015; 44: 674-6.

13. DiCarlo C, Silverman J, Bunker M. Type 1 autoimmune pancreatitis associated with mucinous cystic neoplasm. Am J Clin Pathol 2016; 146(Suppl 1): 290 . 